\section{(6) OPEN ACCESS}

\title{
Exposure to welding fumes is associated with hypomethylation of the F2RL3 gene: a cardiovascular disease marker
}

\author{
Mohammad B Hossain, ${ }^{1}$ Huiqi Li, ${ }^{1}$ Maria Hedmer, ${ }^{1}$ Håkan Tinnerberg, ${ }^{1}$ Maria Albin, ${ }^{1}$ \\ Karin Broberg ${ }^{1,2}$
}

- Additional material is published online only. To view please visit the journal online (http://dx.doi.org/10.1136/ oemed-2015-102884)

${ }^{1}$ Department of Laboratory Medicine, Division of Occupational and Environmental Medicine, Lund University, Lund, Sweden ${ }^{2}$ Unit of Metals \& Health, Institute of Environmental Medicine, Karolinska Institutet, Stockholm, Sweden

\section{Correspondence to} Dr Karin Broberg, Unit of Metals \& Health, Institute of Environmental Medicine, Karolinska Institutet, Nobels Väg 13, Stockholm 171 77, Sweden; karin.broberg@ki.se

Received 10 February 2015 Revised 7 July 2015 Accepted 14 July 2015 Published Online First 22 September 2015

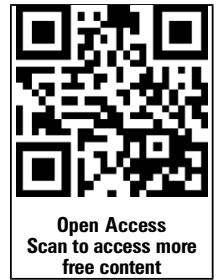

\section{ABSTRACT}

Background Welders are at risk for cardiovascular disease. Recent studies linked tobacco smoke exposure to hypomethylation of the F2RL3 (coagulation factor II (thrombin) receptor-like 3) gene, a marker for cardiovascular disease prognosis and mortality. However, whether welding fumes cause hypomethylation of F2RL3 remains unknown.

Methods We investigated 101 welders (median span of working as a welder: 7 years) and 127 unexposed controls (non-welders with no obvious exposure to respirable dust at work), age range 23-60 years, all currently non-smoking, in Sweden. The participants were interviewed about their work history, lifestyle factors and diseases. Personal sampling of respirable dust was performed for the welders. DNA methylation of F2RL3 in blood was assessed by pyrosequencing of four $\mathrm{CpG}$ sites, CpG_2 (corresponds to cg03636183) to CpG_5, in F2RL3. Multivariable linear regression analysis was used to assess the association between exposure to welding fumes and F2RL3 methylation.

Results Welders had $2.6 \%$ lower methylation of CpG_5 than controls $(p<0.001)$. Higher concentrations of measured respirable dust among the welders were associated with hypomethylation of $\mathrm{CpG} \_2$, $C p G \_4$ and CpG_5 ( $\beta=-0.49$ to $-1.4, p<0.012) ; p<0.029$ adjusted for age, previous smoking, passive smoking, education, current residence and respirator use. Increasing the number of years working as a welder was associated with hypomethylation of $\mathrm{CpG} \_4$ (linear regression analysis, $\beta=-0.11, p=0.039$, adjusted for previous smoking). Previous tobacco smokers had $1.5-4.7 \%$ $(p<0.014)$ lower methylation of 3 of the $4 \mathrm{CpG}$ sites in F2RL3 (CpG_2, CpG_4 and CpG_5) compared to neversmokers. A non-significant lower risk of cardiovascular disease with more methylation was observed for all CpG sites.

Conclusions Welding fumes exposure and previous smoking were associated with F2RL3 hypomethylation. This finding links low-to-moderate exposure to welding fumes to adverse effects on the cardiovascular system, and suggests a potential mechanistic pathway for this link, via epigenetic effects on F2RL3 expression.

\section{INTRODUCTION}

Exposure to welding fumes is associated with an increased risk of cardiovascular disease (CVD); the standardised incidence ratio for acute myocardial infarction was $1.12(95 \%$ CI 1.01 to 1.24$)$ in a

\section{What this paper adds}

- Exposure to welding fumes has been reported to be associated with increased risk of cardiovascular disease. Tobacco smoke exposure has recently been linked to hypomethylation of the F2RL3 gene, a marker for mortality and cardiovascular disease prognosis. Both welding fumes and tobacco smoke consist mainly of ultrafine condensation particles.

- We investigated F2RL3 methylation by pyrosequencing in welders and controls.

- We found that exposure to welding fumes was associated with F2RL3 hypomethylation, suggesting evidence that low-to-moderate exposure to welding fumes may have adverse effects on the cardiovascular system via epigenetic modifications.

Danish prospective study of welders followed until $2006,{ }^{1}$ and the standardised mortality ratio for ischaemic heart disease was 1.35 (95\% CI 1.1 to 1.6) in a Swedish study of welders followed until 1995. ${ }^{2}$ Despite these observational data, the mechanisms coupling exposure to welding fumes with harmful cardiovascular events remain unclear.

Low DNA methylation, so-called hypomethylation, of the F2RL3 gene in blood is a predictor of mortality from CVDs and cancers. ${ }^{3}$ F2RL3 hypomethylation can also serve as a marker of CVD prognosis. ${ }^{4}$ Recent studies have found that tobacco smoking, a strong risk factor for CVD, is associated with hypomethylation of F2RL3. ${ }^{5-8}$ Breitling et al first reported in a population-based epidemiological study that $\operatorname{cg} 03636183$ of F2RL3 (CpG_2) was significantly hypomethylated in smokers. Later studies have shown that, along with $\mathrm{CpG}_{-} 2, \mathrm{CpG}_{-} 4$ and CpG_5 can serve as biomarkers for current and lifetime smoking, ${ }^{9}$ and as strong predictors of CVD-associated mortality. ${ }^{3}$ F2RL3 is expressed in different cell types, including circulating leucocytes, ${ }^{10}$ and encodes thrombin protease-activated receptor 4 (PAR-4), a cell surface protein. ${ }^{11}$ PAR-4 functions in blood coagulation ${ }^{12}$ and activation of PAR-4 is important for multiple aspects of immune function, including recruitment of leucocytes, modulation of rolling and adherence of neutrophils 
and eosinophils, as well as regulation of vascular endothelial cell activity. ${ }^{10} \quad 12-14$ These physiological events also occur as early steps in inflammatory reactions in the vascular system, ${ }^{10} 1215$ and moreover, these events have been observed to occur more frequently in smokers and partly in relation to welding particle exposures. $^{16-19}$

We hypothesised that welding fume exposure is associated with hypomethylation of $F 2 R L 3$, and we analysed the methylation status of F2RL3 in a group of welders and non-exposed controls.

\section{MATERIALS AND METHODS Study population}

We enrolled 101 welders from 10 different companies in southern Sweden. The companies were medium sized and produced heavy vehicles, lifting tables, stoves, heating boilers and pumps, and equipment for the mining industry. Detailed characteristics of 8 of the 10 companies which employed 83 welders were

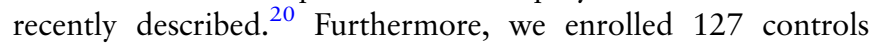
from seven different companies: participants from six companies were 'blue-collar' worker with the routine task of organising grocery goods and participants from the seventh company were working as gardeners; these unexposed control workers had no obvious exposure to respirable dust at the workplace. All study participants were male and currently non-smokers. Among the previous smokers, one reported smoking in the past 12 months; the remaining previous smokers stopped smoking at least 1 year before study enrolment. Structured questionnaire-based interviews were carried out by a trained nurse to obtain information about age, height and weight (all coded as continuous variables), ethnicity (participants and their parents' nationality), education (five categories: primary school, high school, professional school, university $<3$ years, university $>3$ years of education), medical history, personal and family disease history (cancer and CVDs), diet (frequency of intake of fruit, vegetables and fish), physical activity (four levels, from sedentary to regular exercise), previous smoking history (yes or no; if yes, year of start and end), passive smoking (at home and/or at work), alcohol consumption (wine or other alcohol consumption, with six different levels in each), current residence, having a wood burning stove or boiler at home, exposure to wood smoke from the neighbourhood, exposure to traffic (traffic intensity around the residence and time spent in traffic every day), working environment, occupational history, and hobbies with exposure to smoke (eg, working with car engines, etc). The participants were asked whether they had had myocardial infarction, angina pectoris, hypertension, stroke, thrombosis or other CVDs diagnosed by a physician. All study participants answered the same questionnaire, apart from questions regarding work tasks that differed between welders and controls.

Venous blood samples were collected from the participants. This study was approved by the Regional Ethical Committee of Lund University, Sweden, and all study participants gave their informed written consent to take part in the study.

\section{Occupational exposure assessment Monitoring of exposure}

Respirable dust was measured once in each of the welding companies and samples were collected in the breathing zones of welders. For welders who were wearing powered air purifying respirators (PAPRs), air outside the PAPRs was sampled. Exposure to respirable dust was measured by air sampling on pre-weighed $37 \mathrm{~mm}$ mixed cellulose ester filters $(0.8 \mu \mathrm{m}$ pore size) fitted in leak-free cassettes (Sure-Seal). Respirable dust cyclone air samplers of nickel-plated aluminium (BGIL4, BGI Inc) were attached to the filter cassettes. Battery powered sampling pumps (MSA Escort Elf) were operated at a flow rate of 2.2 $\mathrm{L} / \mathrm{min}$. The airflow was checked before, during and after the sampling with a primary calibrator (TSI Model 4199, TSI Inc). Most of the air sampling was performed during full-shift work; the average sampling time was $6.8 \mathrm{~h}$ (range $2.4-8.6 \mathrm{~h}$ ). The filter samples were analysed gravimetrically for respirable dust according to a certified method. The limit of detection (LOD) was $0.05 \mathrm{mg} / \mathrm{sample}$.

Parallel measurements of respirable dust were performed to assess the workplace protection factor for the PAPRs. A setup consisting of two parallel sampling systems for respirable dust was used: one for sampling inside and one for sampling outside the PAPR on the shoulder in the breathing zone. Parallel samplings were performed on three workers at different companies and the respirable dust concentrations were at least three times lower inside the PAPRs compared with concentrations outside in the breathing zones. ${ }^{20}$

The exposure to respirable dust was also measured by personal sampling in the breathing zone for 19 workers from two control companies. The average sampling time for the controls was $7.2 \mathrm{~h}$. Stationary measurements of respirable dust were conducted in four other control companies with a direct-reading instrument (Sidepak Model AM510, TSI Inc). In these six companies, the particle number concentrations (size range 20-1000 nm) were also measured with direct-reading, stationary instruments (P-Trak, TSI Inc).

\section{Exposure assessment of welding fumes}

Respirable dust was measured for 53 of 101 welders. For the remaining 48 welders without measurements of respirable dust, their exposure to welding fumes was assessed from exposure data of the 53 welders aforementioned and 17 welders working with similar tasks at the same companies, but not included in the study, as well as exposure data from a previous study. ${ }^{19} \mathrm{We}$ excluded two participants from the exposure assessment: one welder reported that he worked only with soldering/brazing, which seemed unreasonable and one welder's information about the local exhaust ventilation was missing, making it difficult to correctly estimate his exposure. After excluding these two participants, the total number of welders with data on estimated respirable dust exposure was $n=99$. In order to calculate the exposure to respirable dust for welders with PAPRs, the respirable dust concentrations were reduced by a correction factor of three to get a better estimate of the exposure inside the PAPRs. The correction factor was based on the results from our parallel respirable dust measurements described above, and literature data on the workplace protection factor of PAPRs. ${ }^{20-23}$

\section{Analysis of DNA methylation}

DNA was isolated from venous blood with the QIAamp DNA Blood Midi kit (Qiagen, catalogue nr 51183). The DNA quality was evaluated on a NanoDrop spectrophotometer (Thermo Scientific, NanoDrop 1000) and the DNA showed good quality $(260 / 280 \mathrm{~nm}>1.80)$. DNA was bisulfite treated with the EZ DNA Methylation kit (Zymo Research, catalogue nr D5008).

Pyrosequencing assays were designed to quantify the percentage of methylation of F $2 R L 3$ at $\mathrm{CpG}$ sites that have previously been linked to tobacco smoke. ${ }^{5}$ F2RL3 is on chromosome 19 and has two exons. The cg03636183 site (number based on Illumina $27 \mathrm{~K}$ and $450 \mathrm{~K}$ beadchips) is located on a $\mathrm{CpG}$ island (http://www.ncbi.nlm.nih.gov/epigenomics/genome/GCF 000001405.13/gene:9002/) in exon two. The cg03636183 site 
(labelled CpG 2), based on previous studies ${ }^{3} 924$ and three other $\mathrm{CpG}$ sites downstream (labelled $\mathrm{CpG}_{-} 3$ to $\mathrm{CpG}_{-} 5$ ) within a total length of $70 \mathrm{bp}$ were analysed. Two pyrosequencing assays covered these four sites: the first assay (amplicon length of 235 nucleotides) encompassed one site and the second assay (amplicon length of 341 nucleotides) encompassed three sites (see online supplementary table S1). The assays were designed by PyroMark Assay Design 2.0 software (Qiagen). The forward primers were biotinylated. PCR was performed using PyroMark PCR reagents (Qiagen, catalogue nr 972807). The PCR product was purified using Streptavidin Sepharose High Performance beads (Amersham Biosciences, catalogue nr 17-5113-01). Pyrosequencing was carried out using the PSQ HS96 Pyrosequencing System (Qiagen). We repeated 21\% (N=48) of the samples and found the variation in coefficients (VC) as $5.4 \%, 3.5 \%, 2.5 \%$ and $3.3 \%$ for CpG_2 to CpG_5, respectively. Negative controls were included in each run.

\section{$C$ reactive protein and serum amyloid $A$ measurements}

$C$ reactive protein (CRP) was measured in plasma by immunoturbidimetry, and serum amyloid A (SAA) was measured in serum by immunonephelometry at the Department of Clinical Chemistry in Lund University Hospital using standard protocols. $^{25}$

\section{Statistical analyses}

Differences in characteristics and F2RL3 methylation percentages between welders and controls were compared by the Mann-Whitney U test for continuous variables and Fisher's exact test for categorical variables.

General linear models were employed to explore the main research hypothesis, that is, that working as a welder is associated with F2RL3 methylation, as well as that measured/calculated respirable dust exposure and years working as a welder are associated with F2RL3 methylation. In addition, the general linear model was employed to evaluate associations between previous smoking and F2RL3 methylation. Associations between F2RL3 methylation and CVD were investigated by logistic regression. The methylation levels observed at the $\mathrm{CpG}_{-} 4$ were not normally distributed when combining welders and controls; however, it was normally distributed when analysing welders only.

We performed unadjusted, adjusted and full model analyses while investigating the associations between welding versus F2RL3 methylation. Adjusted analyses included age (continuous) and previous smoking (yes or no), as these two variables were reported to be associated with F2RL3 methylation. For identification of additional factors to be included in the full model, we considered variables from both published literature and our own data, including individual characteristics, diet, lifestyle, passive smoking and disease history. These variables were tested individually in our main hypothesis, that is, the differences of F2RL3 methylation between welders and controls. Any variable altering the effect estimate by more than $10 \%$ with at least two of four CpG sites was included in the full model. These additional variables were education (low or high), passive smoking (yes or no) and current residence (big city or others). To be consistent, we included the same variables in the full model investigating the associations between welding and methylation, and when investigating the association between previous smoking and methylation. While investigating the association between F2RL3 methylation and CVD, adjusted analyses included age and body mass index (BMI; continuous). Other possible confounders were considered, but none altered the effect estimate by more than $10 \%$ in the associations between F2RL 3 methylation and CVD.

For the analysis of measured respirable dust versus methylation, the use of a respirator was also considered. When the number of years working as a welder was evaluated, age was not included in the adjusted analysis and full model, as age and years worked were highly correlated $(r=0.75)$, and including both of them in the same model increased the SE by more than $50 \%$ (eg, from 0.032 to 0.049 ). All statistical analyses were performed using SPSS V.22.0 (SPSS Inc) and statistical significance refers to $\mathrm{p}<0.05$ (two tailed).

\section{RESULTS}

Welders were exposed to average levels of $1.2 \mathrm{mg} / \mathrm{m}^{3}$ (range $0.1-19.3 \mathrm{mg} / \mathrm{m}^{3}$ ) of respirable dust, whereas all the control subjects measured had exposure to respirable dust lower than $0.2 \mathrm{mg} / \mathrm{m}^{3}$. Characteristics of study subjects, including methylation of $F 2 R L 3$, are presented in table 1.

Welders and control subjects did not differ in age, BMI, ethnicity, reported CVD, family history of CVD, number of individuals who reported previous smoking, or hobbies with exposure to dust, gases and fumes. Furthermore, welders and control subjects did not differ in intake of vegetables, fruit or fish, use of snus, consumption of wine, or time spent in traffic (not in table 1). The welders were likely to have shorter education, live in smaller cities and have higher exposure to passive smoking and to respirable dusts compared to controls. $\mathrm{CpG}_{-} 2, \mathrm{CpG}_{-} 4$ and $\mathrm{CpG}_{-} 5$ were significantly correlated with each other (Pearson, $r_{s}=0.24-0.64$ ). Welders had a significantly lower methylation of $\mathrm{CpG}_{-} 5$ and higher methylation of $\mathrm{CpG} 3$ than controls (table 1).

In linear regression analysis, welders showed a significantly lower methylation of $\mathrm{CpG} 5$ compared to controls, also after adjustment for age and previous smoking (table 2).

However, after additional adjustments for passive smoking, education and current residence, the associations became nonsignificant $(p=0.061)$. Compared to controls, welders had higher methylation in CpG_2 (only in full model) and CpG 3 (table 2).

Measured ( $\mathrm{N}=53$ welders) and calculated $(\mathrm{N}=99)$ concentrations of respirable dust were associated with DNA methylation (table 3A): higher concentrations of respirable dust (both measured and calculated) were significantly associated with hypomethylation of $\mathrm{CpG}_{-} 2$ and $\mathrm{CpG}_{-} 4$ (figure 1) and (only measured) $\mathrm{CpG}_{-}$5.

The effect estimates were in general higher for the measured values than the calculated values. Adjustments with age, previous smoking, passive smoking, education, current residence and respirator use marginally changed the estimates, but not the statistical significance, except for calculated respirable dust versus CpG 2 (table 3A). Furthermore, we analysed the association between years working as a welder and methylation of F2RL3. Methylation of CpG_4 was inversely associated with working years $(\beta=-0.11, p=0.039$, adjusted for previous smoking; table $3 \mathrm{~B})$, but this association became non-significant in the full model $(\beta=-0.089, p=0.096)$. When age was included in the full model, the associations became statistically non-significant, which is most likely due to overadjustments, as age and working years as welders were highly correlated $\left(r_{s}=0.75\right)$. Both age and working years as welder were associated with $\mathrm{CpG}_{4} 4$ methylation. However, when age and working years were included in the same model, both of them became non-significant, which indicates that the model was suffering from collinearity. In order to distinguish the effect of working years as a welder from the general effect of age on F $2 R L 3$ methylation, we investigated the 
Table 1 Characteristics of welders and controls, and comparisons between groups

\begin{tabular}{|c|c|c|c|}
\hline Characteristics & Welders $(n=101)$ & Controls $(n=127)$ & $p$ Value \\
\hline Age (year) & $41(23-60)$ & $43(23-56)$ & $0.90^{*}$ \\
\hline $\mathrm{BMI}\left(\mathrm{kg} / \mathrm{m}^{2}\right)$ & $27.7(21.4-34.9)$ & $27.1(22.3-34.2)$ & $0.48^{*}$ \\
\hline Ethnicity: European/others (European, \%) † & $96 / 5(95)$ & $122 / 5(96)$ & $0.75 \ddagger$ \\
\hline Low/high education (low, \%)§ & $94 / 7(93)$ & $109 / 17(87)$ & $0.032 \ddagger$ \\
\hline Cardiovascular disease: yes/no (yes, \%)ף & $23 / 78(23)$ & 25/102 (20) & $0.63 \ddagger$ \\
\hline Family history of cardiovascular disease: yes/no (yes, \%)** & $44 / 57(44)$ & $46 / 81(36)$ & $0.28 \ddagger$ \\
\hline Current residence: big city/others (big city, \%)†† & $10 / 91(10)$ & $29 / 98(23)$ & $0.013 \ddagger$ \\
\hline Previous smoking: yes/no (yes, \%) & $43 / 58(43)$ & $43 / 83(34)$ & $0.21 \ddagger$ \\
\hline Passive smoking: yes/no (yes, \%) & $29 / 72(29)$ & $16 / 111(13)$ & $0.0041 \ddagger$ \\
\hline Hobby with smoke: yes/no (yes, \%) & $9 / 92(9)$ & $5 / 122(4)$ & $0.17 \ddagger$ \\
\hline Respirable dust $\left(\mathrm{mg} / \mathrm{m}^{3}\right) \ddagger \ddagger$ & $1.1(0.2-8.3)$ & $0.1(0.0-0.2)$ & $<0.001^{*}$ \\
\hline CpG_2 methylation (\%) & $76.7(71.5-83.0)$ & $76.5(70.3-81.2)$ & $0.27^{*}$ \\
\hline CpG_3 methylation (\%) & $78.5(75.6-81.2)$ & $77.4(74.1-82.7)$ & $<0.001^{*}$ \\
\hline CpG_4 methylation (\%) & $88.4(77.0-94.2)$ & $88.9(78.0-94.2)$ & $0.68 *$ \\
\hline CpG_5 methylation (\%) & $87.1(82.8-97.5)$ & 89.7 (81.8-97.1) & $<0.001^{*}$ \\
\hline
\end{tabular}

Medians (5-95\%) are presented, if not indicated otherwise.

${ }^{*}$ Between welders and controls (Mann-Whitney U test).

tParents of the participants from Europe/others (\%).

‡Between welders and controls (Fisher's exact test).

$\S$ Participants with high school or lower/university education, or higher.

१Participants reported whether they had had myocardial infarction, angina pectoris, hypertension, stroke, blood clot or any other cardiovascular diseases diagnosed by their doctors.

** Participants were asked whether their parents or siblings were diagnosed by doctors for having myocardial infarction, stroke and/or hypertension before they were 65 years old.

††Big city/small city, villages.

$\ddagger \ddagger$ Nineteen of the controls and 53 of the welders were monitored.

BMI, body mass index.

association between age and $\mathrm{CpG}_{-} 4$ in controls as well. The effect estimate was 23\% lower than in the welders, and the $\mathrm{p}$ value was rather close to the significance threshold (data not shown). The difference of the effect of age in welders and in controls suggests that the welders may be influenced by other factors strongly correlated with age, such as working years as a welder. Moreover, in another sensitivity analysis, we also adjusted the models presented in tables 2 and 3A, B with CRP and SAA as markers for acute inflammatory response, but these adjustments did not affect the associations substantially (data not shown).

We also investigated the association between previous smoking and F2RL3 methylation, and F2RL3 methylation and CVD (table 4A, B).

Previous smokers had a significantly lower methylation in CpG_2 (1.5\%), CpG_4 (4.7\%) and CpG_5 (2.1\%) compared to never-smokers. These associations remained significant after adjustments. We found that previous smokers had lower methylation compared with never-smokers both among welders and non-exposed controls, for example, previous smokers showed a $1.6 \%$ lower methylation of $\mathrm{CpG}_{2} 2$ compared to non-smokers in both groups. For $\mathrm{CpG}_{-} 4$, previous smokers showed a $4.0 \%$ and
5.3\% lower methylation in welders and controls, respectively (data not shown). We also performed logistic regression analysis between CpG methylation and self-reported CVD. None of the $\mathrm{CpG}$ sites were significantly associated with history of CVD, although a non-significantly lower risk with more methylation was observed for all $\mathrm{CpG}$ sites.

\section{DISCUSSION}

This study shows that exposure to welding fumes, similar to tobacco smoke, is associated with hypomethylation of $F 2 R L 3$, a risk marker for CVD. The effects on F2RL3 methylation were found at low-to-moderate levels of exposure to welding fumes and suggest that welders might be at risk for CVD, despite precautions taken at lower exposure levels. The welders we studied had a median exposure level of $1.2 \mathrm{mg} / \mathrm{m}^{3}$ welding fumes over a shift, and the occupational exposure limit $(8 \mathrm{~h}$ time weighted average) set by the Swedish Work Environment Authority is $5 \mathrm{mg} / \mathrm{m}^{3} .^{26}$ The fact that welding fumes and tobacco smoke were linked to hypomethylation of F2RL3 suggests that a common factor may cause the observed associations. One common factor is ultrafine particles, and indeed we found strong associations between individual exposure to respirable

Table 2 Association between occupation as a welder and methylation of CpG sites in F2RL3

\begin{tabular}{|c|c|c|c|c|c|c|}
\hline \multirow[b]{2}{*}{ Variable } & \multicolumn{2}{|l|}{ Unadjusted analysis } & \multicolumn{2}{|l|}{ Adjusted analysis } & \multicolumn{2}{|l|}{ Full model } \\
\hline & $\boldsymbol{\beta}(95 \% \mathrm{Cl})$ & p Value & $\boldsymbol{\beta}(95 \% \mathrm{Cl})$ & p Value & $\boldsymbol{\beta}(95 \% \mathrm{Cl})$ & p Value \\
\hline CpG_2 & $0.70(-0.23$ to 1.6$)$ & 0.14 & $0.86(-0.054$ to 1.8$)$ & 0.065 & $1.1(0.094$ to 2.0$)$ & 0.031 \\
\hline CpG_3 & $0.86(0.26$ to 1.5$)$ & 0.0053 & $0.90(0.30$ to 1.5$)$ & 0.0036 & $0.99(0.37$ to 1.6$)$ & 0.0020 \\
\hline CpG_4* & $-0.15(-1.6$ to 1.4$)$ & 0.85 & $0.24(-1.2$ to 1.6$)$ & 0.74 & $0.83(-0.62$ to 2.3$)$ & 0.26 \\
\hline CpG_5 & $-1.8(-3.0$ to -0.59$)$ & 0.0036 & $-1.6(-2.8$ to -0.43$)$ & 0.0079 & $-1.2(-2.4$ to 0.055$)$ & 0.061 \\
\hline
\end{tabular}

The $\beta$ values presented for occupation as a welder versus control are derived from general linear models. Unadjusted analyses: $F 2 R L 3$ methylation=intercept $+\beta$ (occupation); adjusted analyses: $F 2 R L 3$ methylation=intercept $+\beta$ (occupation) $+\beta 1$ (age) $+\beta 2$ (previous smoking); and full model: $F 2 R L 3$ methylation=intercept $+\beta$ (occupation) $+\beta 1$ (age) $+\beta 2$ (previous smoking) $+\beta 3$ (passive smoking) $+\beta 4$ (education) $+\beta 5$ (current residence).

*The residuals for methylation levels for $\mathrm{CpG}_{4} 4$ were not normally distributed when comparing welders and controls and log transformation did not improve the distribution. 
Table 3 (A) Association between respirable dust (measured and calculated) exposure and CpG sites methylation in F2RL3 in welders and (B) linear regression analysis of working years as welder and F2RL3 methylation

\begin{tabular}{|c|c|c|c|c|c|c|c|c|c|c|}
\hline \multicolumn{11}{|l|}{ Panel A } \\
\hline \multirow[b]{3}{*}{ Variable } & \multicolumn{5}{|c|}{ Measured respirable dust $(\mathrm{N}=53)$} & \multicolumn{5}{|c|}{ Calculated respirable dust $(\mathrm{N}=99)$} \\
\hline & \multicolumn{2}{|l|}{ Unadjusted analysis* } & \multicolumn{3}{|c|}{ Full modelt } & \multicolumn{3}{|c|}{ Unadjusted analysis* } & \multicolumn{2}{|l|}{ Full model‡ } \\
\hline & $\boldsymbol{\beta}(95 \% \mathrm{Cl})$ & p Value & \multicolumn{2}{|c|}{$\boldsymbol{\beta}(95 \% \mathrm{Cl})$} & $p$ Value & \multicolumn{2}{|c|}{$\boldsymbol{\beta}(95 \% \mathrm{Cl})$} & p Value & $\boldsymbol{\beta}(95 \% \mathrm{Cl})$ & p Value \\
\hline CpG_2 & $-0.51(-0.85$ to -0.16$)$ & 0.0045 & $-0.49(-0$ & $-0.10)$ & 0.014 & -0.44 & to -0.036$)$ & 0.033 & $-0.38(-0.79$ to 0.025$)$ & 0.065 \\
\hline CpG_3 & $-0.12(-0.31$ to 0.076$)$ & 0.23 & $-0.059(-$ & $0.16)$ & 0.59 & -0.19 & to 0.0057 ) & 0.057 & $-0.17(-0.38$ to 0.039$)$ & 0.11 \\
\hline CpG_4 & $-1.4(-2.0$ to -0.80$)$ & $<0.001$ & $-1.1(-1$ & 46) & 0.0011 & -1.0( & $-0.35)$ & 0.0032 & $-0.71(-1.4$ to -0.062$)$ & 0.032 \\
\hline CpG_5 & $-0.64(-1.1$ to -0.15$)$ & 0.012 & $-0.64(-1$ & $0.069)$ & 0.029 & -0.25 & to 0.32 ) & 0.39 & $-0.089(-0.66$ to 0.48$)$ & 0.76 \\
\hline \multicolumn{11}{|c|}{ Panel B: Working years as welder $(\mathrm{N}=100)$} \\
\hline & \multicolumn{2}{|c|}{ Unadjusted analysis* } & & \multicolumn{4}{|c|}{ Adjusted analysis§ } & \multicolumn{2}{|c|}{ Full model } & \\
\hline Variable & \multicolumn{2}{|c|}{$\boldsymbol{\beta}(95 \% \mathrm{Cl})$} & p Value & \multicolumn{2}{|c|}{$\boldsymbol{\beta}(95 \% \mathrm{Cl})$} & & p Value & \multicolumn{2}{|c|}{$\boldsymbol{\beta}(95 \% \mathrm{Cl})$} & p Value \\
\hline CpG_2 & \multicolumn{2}{|c|}{$-0.027(-0.091$ to 0.037$)$} & 0.41 & \multicolumn{3}{|c|}{$-0.019(-0.082$ to 0.044$)$} & 0.56 & \multicolumn{2}{|c|}{$-0.011(-0.075$ to 0.053$)$} & 0.73 \\
\hline CpG_3 & \multicolumn{2}{|c|}{$-0.020(-0.051$ to 0.011$)$} & 0.20 & \multicolumn{3}{|c|}{$-0.020(-0.051$ to 0.011$)$} & 0.21 & \multicolumn{2}{|c|}{$-0.018(-0.051$ to 0.015$)$} & 0.28 \\
\hline CpG_4 & \multicolumn{2}{|c|}{$-0.13(-0.23$ to -0.021$)$} & 0.019 & \multicolumn{3}{|c|}{$-0.11(-0.21$ to -0.0058$)$} & 0.039 & \multicolumn{2}{|c|}{$-0.089(-0.19$ to 0.016$)$} & 0.096 \\
\hline CpG_5 & \multicolumn{2}{|c|}{$-0.049(-0.14$ to 0.037$)$} & 0.26 & \multicolumn{3}{|c|}{$-0.047(-0.13$ to 0.041$)$} & 0.29 & \multicolumn{2}{|c|}{$-0.033(-0.12$ to 0.055$)$} & 0.46 \\
\hline \multicolumn{11}{|c|}{$\begin{array}{l}\text { The } \beta \text { values are derived from linear regression models. } \\
\text { *Unadjusted analyses: } F 2 R L 3 \text { methylation=intercept }+\beta \text { (respirable dust/calculated respirable dust/years working as welder). } \\
\text { tFull model: intercept }+\beta \text { (respirable dust) }+\beta 1 \text { (age) }+\beta 2 \text { (previous smoking) }+\beta 3 \text { (passive smoking) }+\beta 4 \text { (education) }+\beta 5 \text { (current residence) }+\beta 6 \text { (respirator use). } \\
\text { fFull model: intercept }+\beta \text { (calculated respirable dust) }+\beta 1 \text { (age) }+\beta 2 \text { (previous smoking) }+\beta 3 \text { (passive smoking) }+\beta 4 \text { (education) }+\beta 5 \text { (current residence). For the calculated } \\
\text { respirable dust, use of a respirator was included in the calculation as described in the Materials and methods section. } \\
\S \text { Adjusted analysis: intercept }+\beta \text { (years working as welder) }+\beta 1 \text { (previous smoking). } \\
\text { १Full model: intercept }+\beta \text { (years working as welder) }+\beta 1 \text { (previous smoking) }+\beta 2 \text { (passive smoking) }+\beta 3 \text { (education) }+\beta 4 \text { (current residence) }+\beta 5 \text { (respirator use). }\end{array}$} \\
\hline
\end{tabular}

dust, which in a welding setting predominantly consists of ultrafine particles, and hypomethylation. However, it should be mentioned that other common factors, such as different metals, present in both tobacco smoke and welding fumes, may also cause the association with F2RL3 methylation. We previously reported that welders are exposed to high levels of respirable manganese from welding fumes. ${ }^{20}$ Apart from manganese, iron is also a major component of black steel, and both iron and manganese may adversely affect cardiovascular health. ${ }^{27} 28$

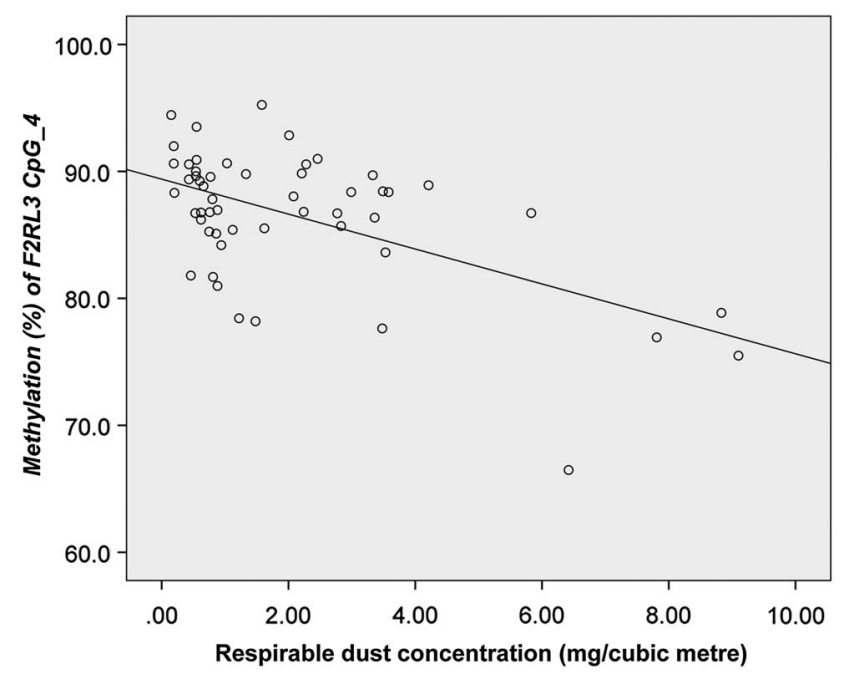

Figure 1 Association between F2RL3 methylation and respirable dust exposure. Scatterplot of F2RL3 CpG_4 methylation (\%) versus measured respirable dust in welders $(n=53)$. The DNA methylation was measured using pyrosequencing in DNA isolated from peripheral blood and exposure to respirable dust was measured by air sampling close to the breathing zone on pre-weighed mixed cellulose ester filters. The fit $\left(R^{2}\right)$ of the line is 0.31 .
F2RL3 is a key gene for recruitment and behaviour of immune cells and blood coagulation. ${ }^{10}{ }^{12-14} 29$ Hypomethylation is often linked to increased gene expression, and if this is the case for F2RL 3, ${ }^{7}$ increased expression of this gene could result in increased inflammation and possibly coagulation. Zhang et $a l^{9}$ reported that hypomethylation of the $\mathrm{CpG}_{-} 4$ site was most strongly associated with smoking behaviour, with an average difference of $5 \%$ between previous smokers and never-smokers, which is in accordance with what we found in our study. We also found that hypomethylation of CpG_4 had the strongest association with exposure to respirable dust, indicating that this particular $\mathrm{CpG}$ site is a main target for the effect of different types of exposure to small particles. The other CpGs showed less consistent associations with occupational exposure to welding fumes. More studies on other types of occupational and environmental particle exposure and methylation of F2RL3 are warranted to identify if aberrant methylation of this gene is a common change in response to exposure to ultrafine particles and, further, the effects of this altered methylation on F2RL3 expression and the cardiovascular system.

Moreover, this study identified significant associations between reported CVD and hypomethylation of sites in F2RL3, despite the fact that the study was rather small and crosssectional. These findings further support the link between this gene and CVD.

Previous publications did not report results for $\mathrm{CpG}_{3} 3$, since the methylation of this $\mathrm{CpG}$ could not be well characterised by the matrix-assisted laser desorption/ionisation-time-of-flight mass spectrometry (MALDI-TOF) assay used which showed high technical variability. ${ }^{3} 524$ However, the pyrosequencing assay we developed identified CpG_3 methylation reliably (VC $3.5 \%$ for repeated tests). Surprisingly, we found that welders showed hypermethylation of $\mathrm{CpG}_{-} 3$ compared to the nonexposed controls, but we did not find any association of $\mathrm{CpG}_{-} 3$ methylation with previous smoking or CVD prevalence. This could indicate that this site is specific for exposure to welding 
Table 4 (A) Association between previous smoking and CpG sites methylation in F2RL3 in welders and controls ( $N=228)$ and (B) Association between F2RL3 methylation and cardiovascular disease by logistic regression $(\mathrm{N}=228)$

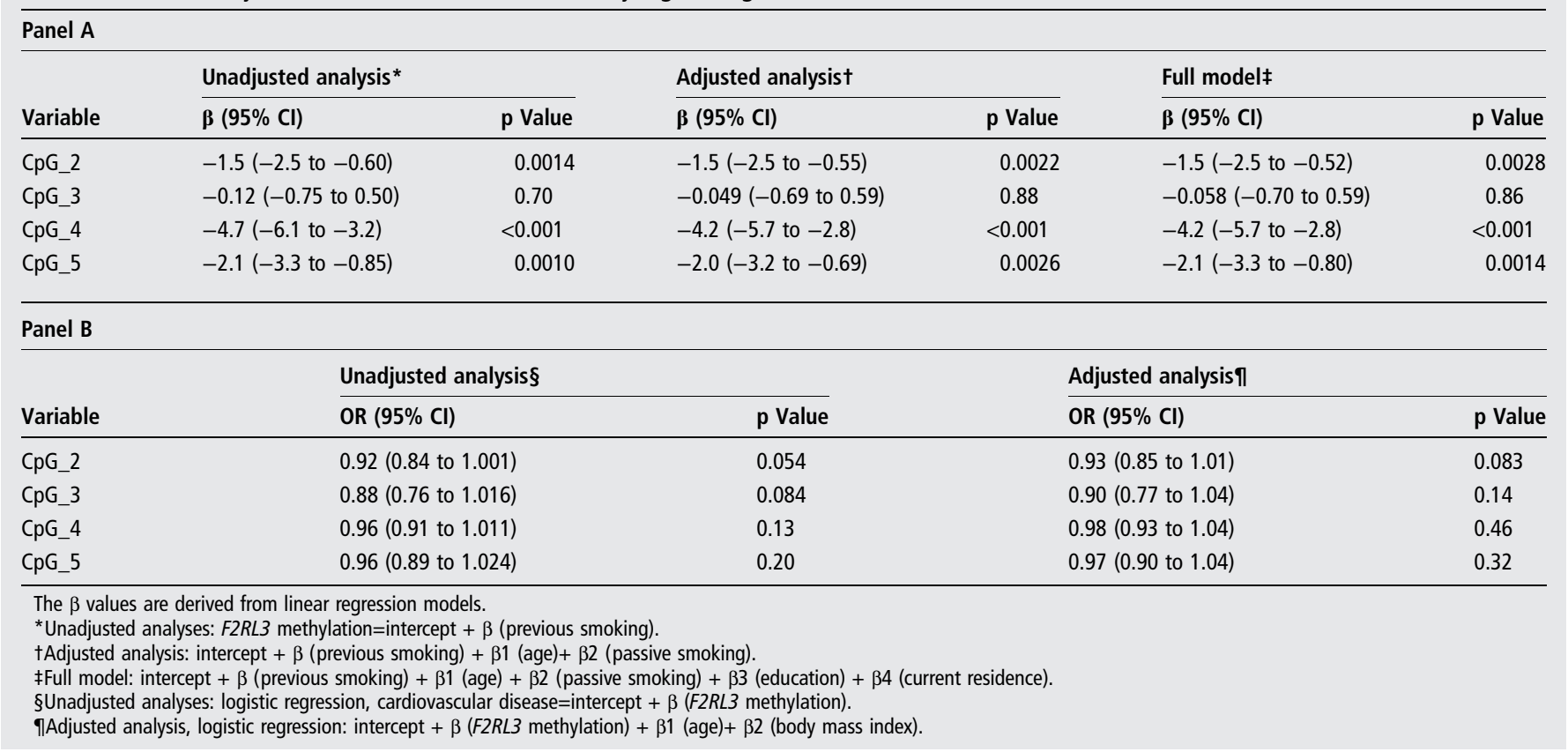

fumes. However, we did not find any association of CpG_3 methylation with welding fume exposure or length of time working as a welder, suggesting that this is unlikely. The significance of hypermethylation of $\mathrm{CpG}_{-} 3$ in welders needs further investigation.

A major advantage of this study was the individual measurements of exposure to welding fumes in a large group of workers, and estimated exposure for the rest of the workers. The higher effect estimates in the analyses restricted to workers whose exposure was individually monitored indicate that this substantially reduced exposure misclassification. A further strength of this study was that the participants were currently non-smokers, as this avoids any masking effect of current smoking on F2RL3 methylation observed in previous studies. ${ }^{6} 9$ Indeed, previous smoking was here associated with hypomethylation of $\mathrm{CpG}_{-} 2, \mathrm{CpG}_{-} 4$ and $\mathrm{CpG}_{-}$5, and this was adjusted for in the regression models. Moreover, we gathered detailed data about other potentially influential factors. However, this study also had some limitations. CpG methylation was measured in the DNA isolated from peripheral blood and not from individual cell populations. DNA methylation patterns may vary among different blood cell types, ${ }^{30}$ and we cannot rule out the possibility that the observed effect on DNA methylation was due to the differences in cell populations. However, we did adjust our data for acute phase response proteins in blood (CRP and SAA), as a proxy for alterations of blood cell composition due to infection or inflammation, but these sensitivity analyses did not change the effect estimates or $\mathrm{p}$ values substantially. Metal fume fever, an acute response to exposure to freshly generated and relatively high concentrations (320-580 mg zinc $/ \mathrm{m}^{3}$ ) of metal-rich particles, ${ }^{31}$ might potentially influence our results by altering the cell composition in blood. Although the welders in our study who were exposed to low-to-moderate levels of welding fumes did not report any fever, and the cytokine levels in welders were mostly similar to the levels in the controls, ${ }^{25}$ we cannot rule out the possibility that the epigenetic effects observed were due to subtle differences in the subpopulation of immune cells caused by particle exposure. The study design is cross-sectional, which limits the conclusions with regard to causality of the observed associations. Some workers used protective devices to protect against inhalation of smoke and we could not measure the respirable dust inside the respirators. Therefore, the measured respirable dust could overestimate the true exposure to inhalable particles. Use of protective masks was, however, adjusted for in the models and this did not change the results significantly. Self-reporting of the personal and family history of CVD was also a limitation of our study and might have blurred the association between F2RL3 methylation and history of CVD. There is an issue of multiple comparisons, as there were four different $\mathrm{CpG}$ sites in the linear regression analysis and both measured and calculated data on respirable dust. However, the CpG sites were partly correlated (ie, they were not independent), and therefore we did not make corrections for multiple testing.

\section{CONCLUSION}

We identified that low-to-moderate levels of exposure to welding fumes were consistently associated with hypomethylation of F2RL3. With the observed similarity of the effects of tobacco smoke and welding fumes on F2RL3, one can hypothesise that ultrafine particles are the causative agents. Our finding suggests a potential mechanistic pathway for the link between welding fumes and CVDs via possible epigenetic effects on F2RL3 expression.

Acknowledgements The study was funded by The Swedish Council for Working Life and Social Research. The authors would like to thank Eva Assarsson for recruiting the study participants. They would also like to thank the Department of Oncology, Lund University, for technical assistance in epigenetic analysis.

Contributors MBH performed the epigenetic and statistical analyses and drafted the paper. $\mathrm{HL}$ recruited the study participants and performed statistical analyses. $\mathrm{MH}$ performed the exposure measurement and assessment. HT and MA contributed to the study design and $\mathrm{HT}$ supervised the exposure measurement and assessment. KB designed and supervised the project.

Competing interests None declared. 
Patient consent Obtained.

Ethics approval Regional Ethical Committee of Lund University, Sweden.

Provenance and peer review Not commissioned; externally peer reviewed.

Open Access This is an Open Access article distributed in accordance with the Creative Commons Attribution Non Commercial (CC BY-NC 4.0) license, which permits others to distribute, remix, adapt, build upon this work non-commercially, and license their derivative works on different terms, provided the original work is properly cited and the use is non-commercial. See: http://creativecommons.org/ licenses/by-nc/4.0

\section{REFERENCES}

1 Ibfelt E, Bonde JP, Hansen J. Exposure to metal welding fume particles and risk for cardiovascular disease in Denmark: a prospective cohort study. Occup Environ Med 2010;67:772-7.

2 Sjogren B, Fossum T, Lindh $\mathrm{T}$, et al. Welding and ischemic heart disease. Int J Occup Environ Health 2002;8:309-11.

3 Zhang $Y$, Yang R, Burwinkel B, et al. F2RL3 methylation in blood DNA is a strong predictor of mortality. Int J Epidemiol 2014;43:1215-25.

4 Breitling LP. Current genetics and epigenetics of smoking/tobacco-related cardiovascular disease. Arterioscler Thromb Vasc Biol 2013;33:1468-72.

5 Breitling LP, Yang R, Korn B, et al. Tobacco-smoking-related differential DNA methylation: 27K discovery and replication. Am J Hum Genet 2011;88:450-7.

6 Wan ES, Qiu W, Baccarelli A, et al. Cigarette smoking behaviors and time since quitting are associated with differential DNA methylation across the human genome. Hum Mol Genet 2012;21:3073-82.

7 Shenker NS, Polidoro S, van Veldhoven K, et al. Epigenome-wide association study in the European Prospective Investigation into Cancer and Nutrition (EPIC-Turin) identifies novel genetic loci associated with smoking. Hum Mol Genet 2013;22:843-51.

8 Sun YV, Smith AK, Conneely KN, et al. Epigenomic association analysis identifies smoking-related DNA methylation sites in African Americans. Hum Genet 2013;132:1027-37.

9 Zhang Y, Yang R, Burwinkel B, et al. F2RL3 methylation as a biomarker of current and lifetime smoking exposures. Environ Health Perspect 2014;122:131-7.

10 Vergnolle N, Derian CK, D'Andrea MR, et al. Characterization of thrombin-induced leukocyte rolling and adherence: a potential proinflammatory role for proteinase-activated receptor-4. J Immunol 2002;169:1467-73.

$11 \mathrm{Xu}$ WF, Andersen $\mathrm{H}$, Whitmore TE, et al. Cloning and characterization of human protease-activated receptor 4. Proc Natl Acad Sci USA 1998;95:6642-6.

12 Leger AJ, Covic L, Kuliopulos A. Protease-activated receptors in cardiovascular diseases. Circulation 2006;114:1070-7.
13 Gomides LF, Duarte ID, Ferreira RG, et al. Proteinase-activated receptor-4 plays a major role in the recruitment of neutrophils induced by trypsin or carrageenan during pleurisy in mice. Pharmacology 2012;89:275-82.

14 Kataoka H, Hamilton JR, McKemy DD, et al. Protease-activated receptors 1 and 4 mediate thrombin signaling in endothelial cells. Blood 2003;102:3224-31.

15 Steinhoff M, Buddenkotte J, Shpacovitch V, et al. Proteinase-activated receptors: transducers of proteinase-mediated signaling in inflammation and immune response. Endocr Rev 2005:26:1-43.

16 Leone A. Smoking, haemostatic factors, and cardiovascular risk. Curr Pharm Des 2007;13:1661-7.

17 Rahman MM, Laher I. Structural and functional alteration of blood vessels caused by cigarette smoking: an overview of molecular mechanisms. Curr Vasc Pharmacol 2007:5:276-92.

18 Jarvela M, Kauppi $\mathrm{P}$, Tuomi $\mathrm{T}$, et al. Inflammatory response to acute exposure to welding fumes during the working day. Int I Occup Med Environ Health 2013;26:220-9.

19 Fang SC, Eisen EA, Cavallari JM, et al. Acute changes in vascular function among welders exposed to metal-rich particulate matter. Epidemiology 2008;19:217-25.

20 Hedmer M, Karlsson JE, Andersson U, et al. Exposure to respirable dust and manganese and prevalence of airways symptoms, among Swedish mild steel welders in the manufacturing industry. Int Arch Occup Environ Health 2014;87:623-34.

21 Goller JW, Paik NW. A comparison of iron oxide fume inside and outside of welding helmets. Am Ind Hyg Assoc J 1985;46:89-93.

22 Han DH. Correlations between workplace protection factors and fit factors for filtering facepieces in the welding workplace. Ind Health 2002;40:328-34.

23 Janssen LL, Nelson TJ, Cuta KT. Workplace protection factors for an N95 filtering facepiece respirator. J Occup Environ Hyg 2007;4:698-707.

24 Breitling LP, Salzmann K, Rothenbacher D, et al. Smoking, F2RL3 methylation, and prognosis in stable coronary heart disease. Eur Heart J 2012;33:2841-8.

25 Li H, Hedmer M, Kåredal M, et al. A cross-sectional study of the cardiovascular effects of welding fumes. PLOS ONE 2015;10:e0131648.

26 Occupational Exposure Limit Values, The Swedish Work Environment Authority's provisions and general recommendations on occupational exposure limit values. AFS 2011: 18: The Swedish Work Environment Authority 2011.

27 Jiang $Y$, Zheng W. Cardiovascular toxicities upon manganese exposure. Cardiovasc Toxicol 2005;5:345-54.

28 Cheng CF, Lian WS. Prooxidant mechanisms in iron overload cardiomyopathy. Biomed Res Int 2013;2013:740573.

29 McDougall JJ, Zhang C, Cellars L, et al. Triggering of proteinase-activated receptor 4 leads to joint pain and inflammation in mice. Arthritis Rheum 2009;60:728-37.

30 Wu HC, Delgado-Cruzata L, Flom JD, et al. Global methylation profiles in DNA from different blood cell types. Epigenetics 2011;6:76-85.

31 Greenberg MI, Vearrier D. Metal fume fever and polymer fume fever. Clin Toxicol (Phila) 2015;53:195-203. 